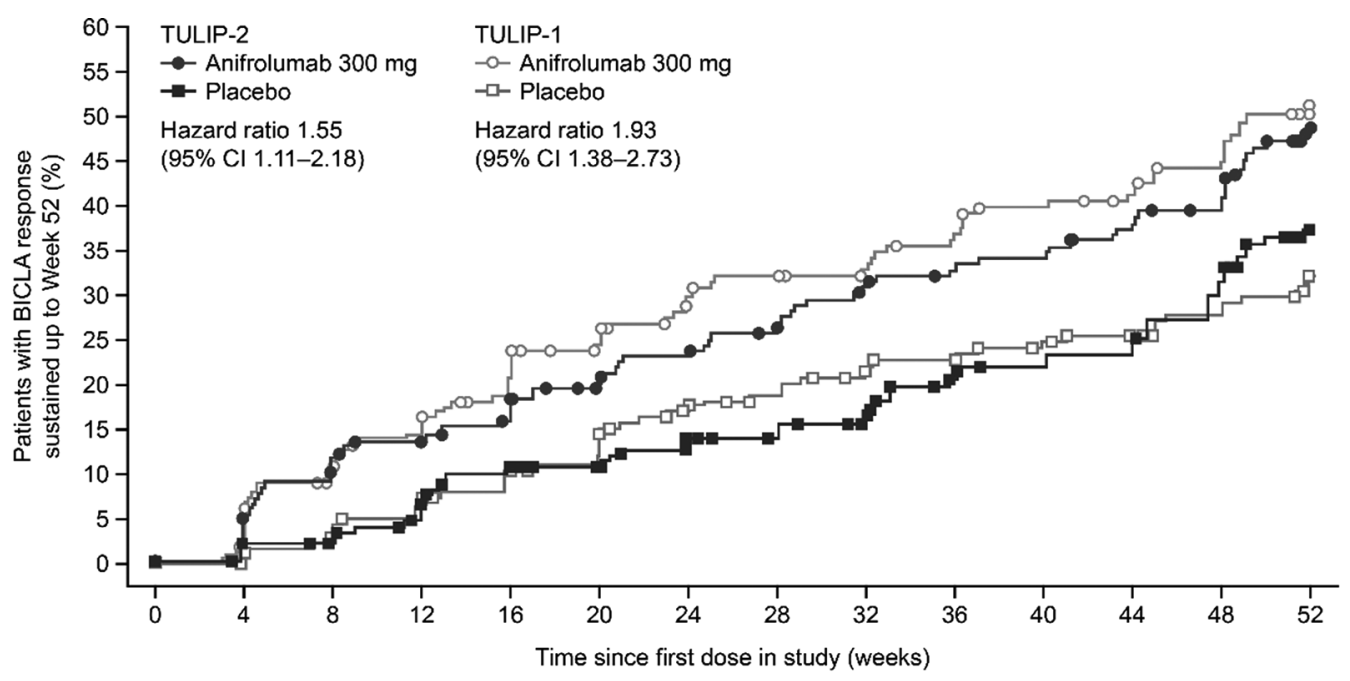

\begin{tabular}{|c|c|c|c|c|c|c|c|c|c|c|c|c|c|c|}
\hline \multicolumn{15}{|c|}{ Number at risk in TULIP-2 } \\
\hline Anifrolumab $300 \mathrm{mg}$ & 180 & 178 & 158 & 150 & 143 & 130 & 124 & 115 & 107 & 101 & 99 & 91 & 85 & 60 \\
\hline Placebo & 182 & 175 & 170 & 160 & 146 & 139 & 132 & 124 & 119 & 105 & 99 & 96 & 85 & 56 \\
\hline \multicolumn{15}{|c|}{ Number at risk in TULIP-1 } \\
\hline Anifrolumab $300 \mathrm{mg}$ & 180 & 170 & 153 & 142 & 129 & 119 & 108 & 102 & 99 & 91 & 85 & 81 & 74 & 53 \\
\hline Placebo & 184 & 179 & 171 & 163 & 154 & 147 & 133 & 127 & 120 & 116 & 110 & 106 & 101 & 83 \\
\hline
\end{tabular}

BICLA, British Isles Lupus Assessment Group-based Composite Lupus Assessment.

Note: Evaluated using a Cox proportional hazards model; time course of sustained BICLA response is not multiplicity adjusted.

Patients without a BICLA response sustained up to Week 52 are censored at the date of study treatment discontinuation or Week 52 ,

whichever occurred earlier. Points on graph show censored patients. Data were analyzed for TULIP-1 data using the amended restricted medication rules.

Abstract P186 Figure 1 Time to onset of BICLA response that was sustained from attainment through week 52 in TULIP-2 and TULIP-1

response from onset through Week 52 in TULIP-2 (anifrolumab, $\mathrm{n}=86$ [47.8\%]; placebo, $\mathrm{n}=57$ [31.3\%]) and in TULIP-1 (anifrolumab, $\mathrm{n}=85$ [47.2\%]; placebo, $\mathrm{n}=55$ [29.9\%]) favored anifrolumab $(\mathrm{HR}=1.55,95 \%$ CI $1.11-2.18$ and $\mathrm{HR}=1.93$, 95\% CI 1.38-2.73, respectively; figure 1).

Conclusions Anifrolumab resulted in numerically favorable differences in BICLA responses maintained through Week 52, and in time to onset thereof, across TULIP studies. These data support the sustainability of clinical benefit with anifrolumab treatment in patients with active SLE.

\section{P187 SYSTEMIC LUPUS ERYTHEMATOSUS IN MALE - ABOUT 5 OBSERVATIONS}

Benamer Mustapha. Faculty of Medicine, University of Algiers, Algiers, Algeria

10.1136/lupus-2020-eurolupus.229

Introduction The occurrence of systemic lupus erythematosus (SLE), an autoimmune disease, is rare in male. It is observed in only $10 \%$ of cases. Its clinical presentation is different, and the evolution is more serious.

Patient presentation The 5 patients are aged 25 years on average. The diagnosis of LES meets the ACR criteria. The mode of revelation is pulmonary embolism, thrombosis of the lower limbs, seritis (pericardial and peritoneal sheet) and cerebral venous thrombosis. These patients have in common a massive proteinuria revealed by an oedema of the lower limbs and where the PBR shows a lupus nephropathy stage III to V. The clinical picture was completed by a characteristic rash, and biologically, a normal normocyte normo-chrome anaemia,
NAA, AC anti-native DNA and antiphospholipid-positive Ac. The treatment is based on the infusions of methylprednisolone and immunosuppressants.

Discussion In all the series, the predilection of LES for women and its rarity in men is noted. The mode of revelation seems more serious in men, but the rarity of joint damage and the constancy of severe glomerular damage were found. Biologically, there is no difference. The use of immunosuppressive drugs is essential in view of the seriousness of the modes of revelation and the aggressiveness of the glomerular damage.

Conclusion Our presentation confirms the rarity of LES male. It emphasizes the seriousness of clinical expressions and the delicate therapeutic management of these forms.

\section{P188 ASSESSMENT OF DISEASE ACTIVITY AND HEALTH RELATED QUALITY OF LIFE IN PATIENTS WITH SYSTEMIC LUPUS ERYTHEMATOSUS AT KENYATTA NATIONAL HOSPITAL}

Eunice Nyambane, Eugene Genga, Loice Achieng, Omondi Oyoo, Fredrick CF Otieno. Dept. of Clinical Medicine and Therapeutics, University of Nairobi, Nairobi, Kenya

\subsection{6/lupus-2020-eurolupus.230}

Background Systemic lupus erythematosus (SLE) is an autoimmune disorder characterised by inflammation in different organ systems. Disease activity varies from remissions to exacerbations and progression. Health-related quality of life (HRQoL) represents the patients subjective perception of living with the disease and how it affects their physical, emotional 
and social functions. The aim of this study was to assess the impact of disease activity on HRQoL.

Methods This was a cross-sectional descriptive study conducted at Kenyatta National Hospital rheumatology and renal outpatient clinics. 62 patients fulfilling $\geq 4$ Systemic Lupus International Collaborating Clinics Criteria (SLICC) 2012 for classification of SLE were consecutively recruited. 27 patients with overlap syndromes were excluded. Disease activity was assessed by the modified Systemic Lupus Erythematosus Disease Activity Index 2000 (cSLEDAI-2K). HRQoL was evaluated using self-administered LupusQoL with scores ranging from 0 (worst) to 100 (best). HRQoL was correlated with age, disease duration and disease activity. Data analysis was performed on SPSS version 23.

Results The study comprised 60 female patients with mean age $34.7 \pm 11.8$ years. The median disease duration was 36 months and ranged from 1-324 months. Mean cSLEDAI score was $7 \pm 5.2$ and median disease activity score was 7 . Renal involvement occurred in $53.2 \%$.

All domains of LupusQoL were impaired. The mean LupusQoL score was 56\% 24.4 (figure 1). SLEDAI scores inversely correlated with scores of physical health, pain, burden to others, body image and general health. The patients with renal disease had significantly lower QoL compared to other patients. Age and disease duration were positively correlated with QoL. Disease duration was associated with a better QoL in the pain, emotional health and body image domains.

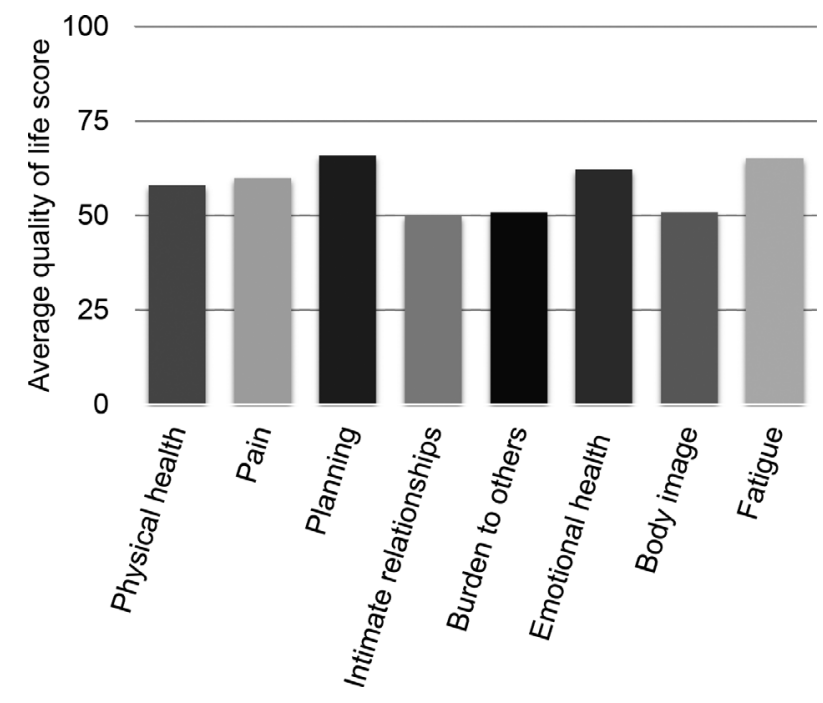

Abstract P188 Figure 1 Quality of life domains

Conclusions Our study showed a low HRQoL in those with active disease. Young age, a recent diagnosis of lupus and presence of renal disease was associated with a poorer QoL.

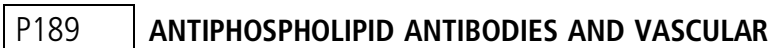 RENAL LESIONS AS PROGNOSTIC FACTORS IN LUPUS NEPHRITIS}

\footnotetext{
${ }^{1}$ Annamaria Paglionico, ${ }^{1}$ Valentina Varriano, ${ }^{2}$ Luca Petricca, ${ }^{1}$ Clara Di Marioa, ${ }^{2}$ Maria Rita Gigante, 'Giacomo Tanti, 'Barbara Tolusso, 'Gianfranco Ferraccioli, 1,2Elisa Gremese. ${ }^{1}$ Division of Rheumatology, Università Cattolica del Sacro Cuore, Rome; ${ }^{2}$ Division of Rheumatology, Fondazione Policlinico Universitario 'A. Gemelli'- I.R.C.C.S., Rome, Italy
}

10.1136/lupus-2020-eurolupus.231
Purpose To determine the role of antiphospholipid antibodies (aPL) and vascular renal lesions on renal prognosis, in terms of time to achieve remission, number of renal flares and development of chronic renal damage in patients with lupus nephritis (LN).

Methods 91 consecutive LN patients have been evaluated and the follow-up data have been collected at the baseline and at $6,12,24$ months and at the last follow-up visit. Histopathological data of 41 patients were evaluated according to the 2016 revision of ISN/RPS classification.

Results Among the $91 \mathrm{LN}$ patients, 31(34.1\%) were aPL positive (aPL +$), 10(32.2 \%)$ of them were affected by Antiphospholipid Antibodies Syndrome (APS), 53.3\% showed a single aPL positivity, $23.1 \%$ double aPL positivity and $15.4 \%$ triple aPL positivity. At the last follow up visit a significant higher number of aPL+ patients showed a persistent complement consumption than aPL negative (aPL-) patients $(p=0.001)$. We observed that aPL- patients showed a remission achievement time slightly earlier than aPL+ patients $(13.6 \pm 1.0$ months vs $16.5 \pm 1.5$ months; log-rank test: $p=0.06$, Breslow test: $\mathrm{p}=0.08$ ) and as expected, patients with a persistent complement consumption achieve remission later (18.2 \pm 1.5 months vs $13.0 \pm 1$ months; log-rank test: $p=0.002$, Breslow test: $\mathrm{p}=0.003)$. Furthermore at the last follow up, a significant higher percentage of aPL+ patients developed persistent proteinuria $(p=0.02)$ and chronic renal failure $(p=0.04)$. Considering histolopathologic features we didn't observe significant differences between aPL+ and aPL- patients but we found two typical vascular lesions (mesangiolysis and vascular thrombi) only in aPL+ patients.

Conclusion Apl positivity is a predictor of worse renal outcome but in our cohort we didn't find an association between aPL positivity and vascular renal lesions at renal biopsy. The worse renal outcome and the late time to achieve remission in aPL+ group can be related to a cumulative vascular damage over time.

\section{P190 A SIMPLIFIED APPROACH FOR PATIENTS WITH SLE TO REPORT DISEASE ACTIVITY USING A REVISED VERSION OF THE SWEDISH SYSTEMIC LUPUS ACTIVITY QUESTIONNAIRE}

${ }^{1,2}$ Susanne Pettersson, ${ }^{1}$ Vera Illescas-Bäckelin, ${ }^{3}$ Andreas Jönsen, ${ }^{1}$ Iva Gunnarsson,
${ }^{4}$ Estelle Trysberg, ${ }^{5}$ Dag Leonard, ${ }^{6}$ Christopher Sjöwall, ${ }^{1}$ Elisabet Svenungsson.
${ }^{1}$ Rheumatology Unit, Dept. of Medicine, Solna, Karolinska Institutet, Karolinska University
Hospital, Stockholm; ${ }^{2}$ Dept. of Neurobiology, Care Sciences and Society, Karolinska
Institutet, Stockholm; ${ }^{3}$ Dept. of Clinical Sciences Lund, Rheumatology, Lund University,
Lund; ${ }^{4}$ Dept. of Rheumatology, Sahlgrenska University Hospital, Göteborg; ${ }^{5}$ Dept. of
Medical Sciences, Science for Life Laboratory, Rheumatology, Uppsala University, Uppsala;
${ }^{6}$ Rheumatology/Division of Neuro and Inflammation Sciences, Dept. of Clinical and
Experimental Medicine, Linköping University, Linköping, Sweden

10.1136/lupus-2020-eurolupus.232

Background/Purpose We compared patients' assessments of SLE disease activity, reported by the SWE-SLAQr, with physicians' assessments using SLE activity measure (SLAM) and SLE disease activity index (SLEDAI-2K).

Methods Patients ( $n=115)$, median age 43 (IQR 24) years, disease duration 15 (IQR 17) years filled out SWE-SLAQr prior to physicians' assessments. Correlations (Spearman's $\rho$ ) were calculated between SWE-SLAQr-total, sub-scales (Symptom score, Patients global) and physicians SLAM, SLEDAI-2K with and excluding the laboratory items, further corresponding items in SLAQ and SLAM were explored. 